\title{
Impact of tuberculosis exposure at home on mortality in children under 5 years of age in Guinea-Bissau
}

\author{
Victor F Gomes, ${ }^{1}$ Andreas Andersen, ${ }^{1}$ Christian Wejse, ${ }^{1,2}$ Ines Oliveira, ${ }^{1}$ Fina J Vieira, ${ }^{3}$ \\ Luis Carlos Joaquim, ${ }^{4}$ Cesaltina S Vieira, ${ }^{3}$ Peter Aaby, ${ }^{1}$ Per Gustafson ${ }^{5}$
}

- An additional table is published online only. To view this file please visit the journa online (http://thorax.bmj.com).

${ }^{1}$ Bandim Health Project, Indepth Network, Bissau, Guinea-Bissau, and Statens Serum Institut, Copenhagen, Denmark

${ }^{2}$ Department of Infectious Diseases, Aarhus University Hospital, Denmark ${ }^{3}$ Hospital de Pneumologia "Raoul Follereau", Bissau, Guinea-Bissau

${ }^{4}$ Hospital Nacional Simao Mendes, Bissau, Guinea-Bissau ${ }^{5}$ Infectious Diseases Research Group, Department of Clinical Sciences, Lund University, Malmö, Sweden

\section{Correspondence to} Victor Francisco Gomes, Projecto de Saúde de Bandim, Apartado 861, 1004 Bissau Codex, Guiné-Bissau; victorfranciscogomes@yahoo. co.uk

Received 27 April 2010

Accepted 11 November 2010 Published Online First

8 December 2010

\section{ABSTRACT}

Objective To assess mortality related to exposure to tuberculosis (TB) at home among children in urban areas of Guinea-Bissau.

Methods In four suburban areas included in the demographic surveillance system of the Bandim Health Project in Bissau, the mortality of children aged $<5$ years living with an adult with TB was compared with the mortality of children in the general population.

Results Children $<5$ years of age exposed to an adult with intrathoracic TB had $66 \%$ higher mortality than unexposed children (HR 1.66, 95\% Cl 1.2 to 2.3). The risk was higher for children living in the same family as a TB case (HR 2.15, 95\% Cl 1.3 to 3.7) than for children living in the same house but not belonging to the same family as the TB case (HR 1.51, 95\% $\mathrm{Cl} 1.0$ to 2.2). For children whose mother had TB, mortality was increased eightfold (HR 7.82, 95\% Cl 2.1 to 30). The risk of death was particularly increased from 6 months following exposure (HR 2.16, 95\% Cl 1.5 to 3.2) and the highest rate of excess mortality was found in children aged $3-4$ years. Excess mortality was highest among children with close contact with an adult with sputum-positive pulmonary TB (HR 1.90, 95\% Cl 1.1 to 3.2), but contact with a sputum-negative case was also associated with increased mortality (HR 1.55, 95\% Cl 1.0 to 2.3). Adjusting for potential confounding factors did not change these results. The mortality among children living in the same houses 3 years earlier was not increased (HR 0.90, 95\% Cl 0.6 to 1.3).

Conclusion Intimate family contact with a TB case represents a significant risk factor for child mortality in a low-income country.

\section{INTRODUCTION}

Child tuberculosis (TB) is still a public health problem. The World Health Organization estimated that at least one million $(11 \%)$ of the nine million annual new cases occur in children. ${ }^{1}{ }^{2}$ In areas with a high incidence of $\mathrm{TB}$, children contribute $15-40 \%$ of the total case load $^{3}$ and experience considerable mortality. ${ }^{4}$ In endemic countries, the focus is mainly on adults with smearpositive $\mathrm{TB}$ while $\mathrm{TB}$ in children receives little attention. ${ }^{4}$ Owing to difficulties with diagnosis, TB is rarely investigated in children in resource-limited settings. ${ }^{5}$

As $\mathrm{TB}$ is an airborne infection, transmission is associated with contact with an infectious $\mathrm{TB}$ case and the intimacy of that contact. ${ }^{67}$ Crowding has been associated with the risk of infection as shared airspace increases exposure to Mycobacterium tuberculosis. $^{6} 89$ TB-related mortality following infection from a smear-positive family source has been reported to be twice as high as for infection from an unknown source. ${ }^{10} 11$ Young children infected with $M$ tuberculosis have a higher risk of developing TB than older children and adults. ${ }^{12}$ In resource-limited settings, TB programmes fail to carry out contact tracing among children exposed to TB. In a study of subjects with a recent tuberculin skin test conversion, children aged $<6$ years developed active TB more frequently than adults $(73 \%$ vs $25 \%) .{ }^{13}$ In resource-limited settings, many deaths in children with pneumonia are TB-related. ${ }^{14}$

Studies on children exposed to $\mathrm{TB}$ are few and data on child mortality are rarely reliable. ${ }^{15} 16$ Using an epidemiological study of adult TB in Bissau between 1996 and 1998, ${ }^{6}$ 17-20 we investigated whether children living with an adult with TB have a higher rate of mortality than children in the general population.

\section{METHODS}

The study was conducted in four districts covered by the Bandim Health Project (BHP), a health and demographic surveillance site in Guinea-Bissau. The population was followed through censuses and information was gathered on socioeconomic factors, sex, ethnicity, birth date, death and migration. Information on deaths was collected every 3 months for children $<3$ years of age.

\section{Identification of adult TB index cases}

Since May 1996 a TB surveillance system, implemented in collaboration with the national TB hospital, identified adult ( $\geq 15$ years) cases of intrathoracic $\mathrm{TB}$ using passive and active case finding. ${ }^{17}$ The procedures have been described previously. ${ }^{17}$ In summary, permanent or temporary adult residents presenting with symptoms of active TB were referred to the TB hospital. Nurses visited the houses of TB cases to detect secondary cases. Patients were examined clinically and interviewed. Microscopic examination of morning sputum was performed on three consecutive days as well as sputum culture and a tuberculin skin test. Chest $\mathrm{x}$-rays were performed and pathological findings were regarded as intrathoracic manifestations. Blood was taken for an HIV test. Patients with one or more positive sputum smears were regarded as smear-positive. Patients with clinical signs of active TB but without a positive bacteriological test were treated with antibiotics and then re-evaluated. ${ }^{21}$ If there was no improvement and suspicion remained, the patient was diagnosed as smear-negative. 


\section{Participants}

Many patients with TB from outside the area sought treatment in Bissau while staying with relatives; these are denoted as guests rather than residents. About 170 cases of TB were registered each year, two-thirds of whom were residents and onethird guests. We assume that the average delay from onset of symptoms to treatment initiation is 3 months. Children living with an adult index case of TB 3 months prior to treatment were considered exposed from this time forward. A child born in the 3-month period prior to treatment was not considered as exposed. As surveillance started on 1 May 1996, we started the follow-up period from 1 February 1996. Due to civil war in June 1998, ${ }^{18}$ follow-up was terminated at the end of May 1998. Follow-up was until 5 years of age. Children acted as controls as long as they were unexposed. Children who were later exposed acted as controls until the day of exposure. The study was retrospective and based on existing data. BCG coverage was high, with $\geq 98 \%$ of the children vaccinated during infancy. ${ }^{22}$

\section{Socioeconomic status}

Socioeconomic status was available from the BHP database. The score $(1-3)$ used divides subjects into three categories (poor, less poor and rich) based on roof type, presence of indoor toilet, electricity and TV, and has been shown to correlate with child mortality. ${ }^{23}$ The information was recorded at birth or at first registration of the child. ${ }^{624}$

\section{Ethical approval}

Analyses were based on general identification of TB cases and data from the demographic surveillance. Ethical approval was therefore not required. The study was retrospective and isoniazid preventive therapy was not policy.

\section{Statistical analysis}

Statistical analyses were conducted using Stata Version 10. The effect of exposure on mortality was evaluated by rate ratios from a Cox analysis with age as the underlying time. The proportional hazards assumption was assessed graphically and tested using Schoenfeld residuals $(p=0.069)$. The borderline nonproportional hazards was met by presenting age-stratified estimates. Robust estimates of standard errors were applied in the results and tables. Correlation between children within houses is conceivable. Adjusting the standard errors for house clustering made almost no difference, changing the robust SE from 0.27 to 0.25 on the estimate of the main exposure effect.

To include hierarchical structures we estimated the exposure effect in each district and estimated a joint estimate while stratifying the baseline mortality hazard by district.

We explored age at exposure and time since exposure started. Exposed children were categorised as $0-11,12-35$ or $36-59$ months of age at the start of exposure. The current time since exposure was grouped as $0-5,6-11$ or $12+$ months since exposure started. Current age was used as the underlying time in the analyses - that is, a child aged 0-11 months at exposure was compared with an unexposed child currently the same age. We conducted subanalyses according to BCG vaccination status (unknown for 9\%). In separate analyses, unknown status was considered as unvaccinated or vaccinated respectively. Neonatal mortality is high. To be exposed a child had to be born and registered at the time of exposure, so only a few children were exposed before 3 months of age. We chose only to follow up the children from the time they reached 3 months of age. If a child was exposed before 3 months of age, he/she was followed up as an exposed individual from 3 months of age.

An adjusted analysis included possible confounders (gender, ethnicity, district, socioeconomic status, schooling of the mother and child crowding). These variables were selected a priori based on previous studies. ${ }^{62324}$ A score for socioeconomic status was calculated adding house indicators (yes $=1 /$ no, missing $=0$ ): corrugated iron roof, electricity, television and indoor toilet. In the adjusted analysis the rare values of 0 and 4 were combined with 1 and 3, respectively. A separate 'missing' category was constructed when information was missing on all four variables. Child crowding was defined as the number of children in the house on 1 April 1997, the midpoint of the examined period. Child crowding was included in the analysis as a linear predictor. Schooling of the mother was categorised as number of years in school (0-3, 4-6, 7-9 and 10+). Information on socioeconomic status and schooling of the mother was available only at time of birth or at first registration.

We examined whether there was excess mortality not related to TB exposure in exposed houses. Mortality was compared between children living in the TB house 3 years before $\mathrm{TB}$ exposure began and children living in the remaining houses. The comparison was made over a period of 2.5 years from February 1993 to the end of May 1995 in order not to overlap with the study period.

\section{RESULTS \\ Mortality among children living in exposed houses compared with the general population}

Two hundred and ninety adult cases of intrathoracic TB were registered in 272 of the 2936 houses with children aged $<5$ years. A total of $4.9 \%(41 / 841)$ of the exposed children died compared with $4.2 \%(526 / 12483)$ of the controls. Table 1 compares mortality between children exposed and not exposed to an adult case of TB in the house. Overall, a $66 \%$ higher mortality was observed in the exposed children (age-adjusted HR 1.66, 95\% CI 1.2 to 2.3). Adjustment was made to examine whether the observed effect was confounded (adjusted HR 1.63, 95\% CI 1.2 to 2.3, table 2). Children known to be BCG vaccinated (83\%) had an $\mathrm{HR}$ of 1.75 (95\% CI 1.3 to 2.4) compared with children

Table 1 Effect of exposure on mortality according to age from February 1996 to June 1998

\begin{tabular}{|c|c|c|c|c|c|c|c|c|}
\hline \multirow{2}{*}{$\begin{array}{l}\text { Age } \\
\text { (months) }\end{array}$} & \multicolumn{3}{|c|}{ Exposed $(N=841)$} & \multicolumn{3}{|c|}{ Unexposed $(N=12483)$} & \multirow[b]{2}{*}{$\operatorname{HR}(95 \% \mathrm{Cl})^{*}$} & \multirow[b]{2}{*}{ HR $(95 \% \mathrm{Cl}) \dagger$} \\
\hline & Deaths & 100 PYO & $\overline{\text { Rate }}$ & Deaths & 100 PYO & $\overline{\text { Rate }}$ & & \\
\hline $3-11$ & 4 & 0.73 & 5.5 & 191 & 28.0 & 6.8 & $0.80(0.3$ to 2.2$)$ & $0.79(0.3$ to 2.1$)$ \\
\hline $12-35$ & 22 & 3.60 & 6.1 & 247 & 65.9 & 3.8 & $1.68(1.1$ to 2.6$)$ & 1.67 (1.1 to 2.6$)$ \\
\hline $36-60$ & 15 & 4.18 & 3.6 & 88 & 57.2 & 1.5 & 2.38 (1.4 to 4.1$)$ & $2.28(1.3$ to 4.1$)$ \\
\hline All & 41 & 8.51 & 4.8 & 526 & 151 & 3.5 & 1.66 (1.2 to 2.3$)$ & 1.63 (1.2 to 2.3$)$ \\
\hline
\end{tabular}

${ }^{*} \mathrm{HR}$ from a model with age as underlying time.

tHR from a model with age as underlying time, adjusted for gender, ethnicity, district, socioeconomic status, schooling of the mother and child crowding.

PYO, person-years of observation. 
Table 2 Adjusted analysis of the overall effect of exposure on mortality from February 1996 to June 1998

\begin{tabular}{|c|c|}
\hline & HR $(95 \% \mathrm{CI})$ \\
\hline Exposure & 1.63 (1.2 to 2.3 ) \\
\hline \multicolumn{2}{|l|}{ Gender } \\
\hline Male & 1 \\
\hline Female & $1.05(0.9$ to 1.2$)$ \\
\hline \multicolumn{2}{|l|}{ Ethnicity } \\
\hline Pepel & 1 \\
\hline Balanta & $0.56(0.4$ to 0.8$)$ \\
\hline Manjaco/Mancanha & $0.91(0.7$ to 1.1$)$ \\
\hline Mandinga/Fula & $0.67(0.5$ to 0.9$)$ \\
\hline Others & $0.81(0.6$ to 1.1$)$ \\
\hline Missing & $2.12(1.3$ to 3.3$)$ \\
\hline \multicolumn{2}{|l|}{ District } \\
\hline Bandim 1 & 1 \\
\hline Bandim 2 & $1.28(1.0$ to 1.6$)$ \\
\hline Belem & $0.90(0.7$ to 1.2$)$ \\
\hline Mindara & $1.06(0.8$ to 1.5$)$ \\
\hline \multicolumn{2}{|l|}{ Socioeconomic score } \\
\hline 1 & 1 \\
\hline 2 & $0.67(0.5$ to 0.9$)$ \\
\hline 3 & $0.58(0.4$ to 0.9$)$ \\
\hline Missing & $0.57(0.4$ to 0.9$)$ \\
\hline \multicolumn{2}{|c|}{ Schooling of mother (years) } \\
\hline $0-3$ & 1 \\
\hline $4-6$ & $0.96(0.8$ to 1.2$)$ \\
\hline $7-9$ & $0.57(0.4$ to 0.8$)$ \\
\hline $10+$ & $0.44(0.2$ to 1.0$)$ \\
\hline Missing & $1.13(0.8$ to 1.5$)$ \\
\hline Crowding & $0.98(0.9$ to 1.0$)$ \\
\hline
\end{tabular}

without BCG vaccination or with unknown BCG status (HR $1.07,95 \%$ CI 0.3 to 3.3). If an unknown BCG status was counted as BCG vaccinated, the estimate of the exposure effect was 1.67 ( $95 \%$ CI 1.2 to 2.3 ). Table 1 in the online supplement shows the results stratified by district. There was an indication of different exposure effect, although this was not significant $(p=0.104)$.

We examined whether the effect in the selected houses was caused by excess mortality unrelated to TB exposure. The mortality among children living in the exposed houses 3 years before exposure was compared with the mortality among children from the general population. The results of the analysis did not indicate any such excess mortality (HR 0.90, 95\% CI 0.6 to 1.3, table 3).

\section{Time since exposure and age at exposure}

Table 4 shows the effect of TB exposure according to time since exposure and age at exposure. During the first 6 months after exposure the observed HR of 1.16 ( $95 \%$ CI 0.7 to 2.0 ) indicated

Table 3 Comparison of mortality rate with children living in the house of a TB case 3 years before exposure starts

\begin{tabular}{|c|c|c|c|c|c|c|c|}
\hline \multirow{2}{*}{$\begin{array}{l}\text { Age } \\
\text { (months) }\end{array}$} & \multicolumn{3}{|c|}{ Exposed $(N=720)$} & \multicolumn{3}{|c|}{ Unexposed $(N=11543)$} & \multirow[b]{2}{*}{ HR $(95 \%$ Cl) } \\
\hline & Deaths & 100 PYO & Rate & Deaths & 100 PYO & Rate & \\
\hline $3-11$ & 6 & 0.70 & 8.6 & 199 & 0.70 & 7.8 & $1.14(0.5$ to 2.5$)$ \\
\hline $12-35$ & 19 & 3.83 & 5.0 & 348 & 3.83 & 5.9 & 0.85 (0.5 to 1.3$)$ \\
\hline $36-60$ & 5 & 3.03 & 1.6 & 87 & 3.03 & 1.8 & 0.91 (0.4 to 2.2 ) \\
\hline All & 30 & 7.56 & 4.0 & 634 & 7.56 & 4.8 & $0.90(0.6$ to 1.3$)$ \\
\hline
\end{tabular}

HR from a model with age as underlying time.

PYO, person-years of observation.
Table 4 HRs (with 95\% Cls) of exposed and unexposed children (exposure stratified by time since exposure and age at exposure)

\begin{tabular}{|c|c|c|c|c|}
\hline \multirow{2}{*}{$\begin{array}{l}\text { Time since } \\
\text { exposure } \\
\text { (months) }\end{array}$} & \multicolumn{3}{|c|}{ Age at exposure (months) } & \multirow[b]{2}{*}{ Total } \\
\hline & $0-11$ & $12-35$ & $36-59$ & \\
\hline $0-5$ & $1.23(0.5$ to 2.8$)$ & $0.96(0.4$ to 2.3$)$ & 1.52 (0.5 to 4.8$)$ & $1.16(0.7$ to 2.0$)$ \\
\hline $6-11$ & $2.22(1.1$ to 4.7$)$ & $1.89(0.8$ to 4.2$)$ & $4.68(1.7$ to 13$)$ & $2.36(1.5$ to 3.8$)$ \\
\hline $12+$ & $0.89(0.2$ to 3.6$)$ & 1.91 (0.8 to 4.6$)$ & $7.18(2.1$ to 24$)$ & $1.88(1.0$ to 3.5$)$ \\
\hline Total & $1.46(0.9$ to 2.5$)$ & $1.46(0.9$ to 2.4$)$ & 3.00 (1.6 to 5.8$)$ & $1.66(1.2$ to 2.3$)$ \\
\hline
\end{tabular}

little effect on survival. A twofold increase in mortality was observed thereafter with HRs of 2.36 (95\% CI 1.5 to 3.8 ) and 1.88 (95\% CI 1.0 to 3.5$)$ at $6-11$ months and $\geq 12$ months after exposure, respectively. Compared with the first 6 months, the combined estimate of 2.16 (95\% CI 1.5 to 3.2) for $>6$ months exposure was significantly different (test of interaction, $\mathrm{p}=0.060$ ). The effect of exposure among 3-4-year-old children was twofold higher than in children aged $<3$ years (HR $3.0(95 \%$ CI 1.6 to 5.8 ) vs HR 1.46 (95\% CI 1.0 to 2.1 ), test of interaction, $\mathrm{p}=0.057$ ).

\section{Proximity, relation and health status of TB cases}

Although the difference was not statistically significant $(p=0.282)$, we observed a higher rate of mortality in children from the same family as the TB case than in children living in the same house but belonging to a different family (table 5). Among the 12 children with a mother with TB, we observed a high mortality rate of 18 per 100 person-years of observation (HR 7.82, 95\% CI 2.1 to 30). Mortality was higher in houses in which the TB case was temporarily staying than in houses where the TB case was a permanent resident (HR 1.92 (95\% CI 1.2 to 3.1$)$ vs HR 1.42 (95\% CI 0.9 to 2.2$)$ ). Marginally higher mortality was observed for smear-positive TB cases than for smear-negative cases (HR 1.90 (95\% CI 1.1 to 3.2) vs HR 1.55 (95\% CI 1.0 to 2.3$)$ ). Severity of disease, measured according to whether the TB case died during treatment, did not influence the effect (table 5).

Table 5 Mortality among exposed children according to proximity to and health status of the adult TB case compared with unexposed children

\begin{tabular}{|c|c|c|c|}
\hline & Rate (deaths/100 PY0) & HR (95\% Cl) & p Value \\
\hline Unexposed & $3.48(526 / 151)$ & 1 & \\
\hline \multicolumn{4}{|l|}{ Proximity in the house } \\
\hline Same family & $5.94(13 / 2.19)$ & $2.15(1.3$ to 3.7$)$ & \multirow[t]{2}{*}{0.28} \\
\hline Different family & $4.43(28 / 6.32)$ & $1.51(1.0$ to 2.2$)$ & \\
\hline \multicolumn{4}{|l|}{ Relation to the TB case } \\
\hline Mother & $17.9(2 / 0.11)$ & $7.82(2.1$ to 30$)$ & \multirow[t]{3}{*}{0.05} \\
\hline Other relative & $4.01(22 / 5.48)$ & $1.42(0.9$ to 2.2$)$ & \\
\hline TB case is a guest & $5.82(17 / 2.92)$ & $1.92(1.2$ to 3.1$)$ & \\
\hline \multicolumn{4}{|c|}{ Smear status of the TB case } \\
\hline Positive & $5.35(15 / 2.81)$ & $1.90(1.1$ to 3.2$)$ & \multirow[t]{2}{*}{0.54} \\
\hline Negative & $4.56(26 / 5.70)$ & $1.55(1.0$ to 2.3$)$ & \\
\hline \multicolumn{4}{|c|}{ HIV status of the TB case } \\
\hline Positive & $5.15(16 / 3.12)$ & $1.78(1.1$ to 2.9$)$ & \multirow[t]{3}{*}{0.76} \\
\hline Negative & $4.71(25 / 5.31)$ & $1.61(1.1$ to 2.4$)$ & \\
\hline Missing & $0(0 / 0.09)$ & - & \\
\hline \multicolumn{4}{|c|}{ Survival status of the TB case } \\
\hline Dead & $4.44(14 / 3.15)$ & 1.58 (0.9 to 2.7$)$ & \multirow[t]{3}{*}{0.79} \\
\hline Alive & $5.06(27 / 5.34)$ & $1.72(1.2$ to 2.5$)$ & \\
\hline Missing & $0(0 / 0.02)$ & - & \\
\hline
\end{tabular}

PYO, person-years of observation. 


\section{DISCUSSION}

\section{Main observations}

We retrospectively compared mortality in children aged $<5$ years exposed to $\mathrm{TB}$ at home with mortality in children with unknown exposure in the general population. The study provided important observations. The rate of mortality was $66 \%$ higher among exposed children; thus, 16 of the 41 deaths in exposed children would have been due to TB exposure. No excess mortality was observed 3 years earlier, which indicates that the increased mortality was not caused by generally higher mortality in the exposed houses.

There was a strong association between exposure time and mortality, with a sevenfold higher mortality 12 months after exposure in the oldest children (table 4). Excess mortality was twofold higher for children in the same family as the TB case and eightfold higher for children of mothers with TB. Mortality was highest in children exposed to a smear-positive adult, but those exposed to a smear-negative TB case also had increased mortality.

\section{Strengths and weaknesses of the study}

During the study period the incidence of TB was high in the area $^{17}$ and retrospective verbal autopsies indicated that very few deaths in the adult population were due to undiagnosed $\mathrm{TB}$. Hence, it is unlikely that many TB cases were missed and children regarded as unexposed are therefore unlikely to have been exposed at home. The population is very mobile and it is possible that some of the unexposed controls may have been exposed elsewhere. This would tend to lower the effect estimated. As the TB programme did not carry out case finding among children, the effect is unlikely to have been modified by TB treatment of exposed children.

A weakness of the study is that autopsies were not performed and it is not possible to know if the cause of death was directly related to TB. Medically certified information for the deaths is not available in Guinea-Bissau. Other sources of data such as post-mortem verbal autopsy interviews to ascribe causes of death have to be used. Due to the war which ended the study, ${ }^{18}$ there were no resources for verbal autopsies to assess how many died with a diagnosis of TB. There are no clinical data on children prior to death, and data on respiratory diseases or malnutrition which affects mortality are not available. It was not possible to adjust for changes in socioeconomic status, and our measure of socioeconomic status is crude with only three levels.

\section{Interpretation and consistency with other findings}

As this is an observational study, the observed association between TB exposure and mortality may be confounded and may not imply a direct causal relationship. Adjustment for potentially confounding factors did not change the estimates. Potential confounders not adjusted for, such as indoor air pollution, could have influenced the estimate. We did attempt to control for socioeconomic status which is an important confounder. However, the available information may not have been adequate for this. If the information was not recorded close to the time of death, any decrease in socioeconomic status or child care associated with parental morbidity may not have been adjusted for at all. Additionally, it is unlikely that the type of roof and the presence of an indoor toilet are sensitive to such changes. On the other hand, we argue for circumstances indicating a causal relationship. There is a wide social network in Guinean families and relatives often provide basic necessities and take care of the children when a parent is ill. This would limit the effect of any morbidity-related decrease in socioeconomic status. That the excess risk increased with age indicates that it is directly or indirectly related to TB exposure. Finally, the excess risk was also observed when the TB case was a guest. We find it harder to designate this effect solely to changes in socioeconomic status; in fact, the effect was higher than in houses where the TB case was a relative other than the mother. Another possible explanation is that TB infection might increase the adult's susceptibility to other infections which can be passed to the children. This cannot be ruled out from the given data. TB-related mortality is caused by interactions with other infections and several of the observed deaths were probably due to causes other than TB. Excess mortality was the same in houses with and without HIV-infected TB cases, and it seems unlikely that the effect is merely due to HIV being common among the children in the houses with TB.

The higher relative risk in children aged 3-5years was surprising as younger children may be more susceptible to $\mathrm{TB}$ and have a more rapid progression of disease with higher mortality. ${ }^{25}$ The finding may be a masking of the TB effect due to high infant mortality, or may be a real effect caused by weaning of the BCG-induced protection or by a longer exposure to TB. It was slightly counterintuitive that the estimate increased among children known to be BCG vaccinated However, when the $9 \%$ with unknown vaccination status were included as BCG vaccinated, the exposure effect was almost identical to the overall estimate.

Results similar to our findings were seen in studies from the pre-chemotherapy era in the USA and UK. ${ }^{10}{ }^{26}$ Other studies have shown that the risk of TB infection is strongly related to the proximity to a smear-positive case of pulmonary TB. ${ }^{6-826-28}$ In our study the overall mortality was $90 \%$ in children exposed to a smear-positive adult compared with $55 \%$ in those exposed to a smear-negative TB case. This finding is similar to that reported in a review of the pre-chemotherapy literature ${ }^{29}$ where a considerably increased risk was conveyed by smear-negative adult cases. Indeed, transmission of $M$ tuberculosis from smear-negative patients is known to contribute significantly to the incidence of disease. $^{30}$

The present study explores an observation indicating increased mortality among children living with an adult TB case. Parallel to these analyses, the authors conducted a prospective study providing isoniazid preventive therapy to exposed children, as recommended by the World Health Organization. The exposed children who received isoniazid preventive therapy in that study had lower mortality than the unexposed control population (unpublished data). This further indicates that the excess risk observed in exposed houses was not predominantly due to decreased socioeconomic status or similar confounding factors.

\section{Implications and conclusion}

This study shows that intimate house contact with an adult case of TB increases the risk of mortality to a considerable extent in children aged $<5$ years. Although the excess mortality was higher when the adult had smear-positive TB, there was also a significantly increased risk among children exposed to smearnegative cases of adult TB. There were about 16 excess deaths among children exposed to TB. It is important to note that excess mortality only started from about 6 months after exposure. Hence, there should be ample time for implementing preventive measures.

The directly observed treatment strategies recommended by the World Health Organization should be combined with routine screening of children in contact with adult patients with 
TB. The increased risk of mortality over time suggests that repeated examinations may be warranted. In areas with a high incidence of $\mathrm{TB}$, the feasibility and cost-effectiveness of isoniazid preventive therapy for children aged $<5$ years in contact with an adult with pulmonary TB should be evaluated.

Acknowledgements The authors thank the Bandim Health Project staff in Guinea-Bissau and Denmark for excellent collaboration.

Funding The Swedish International Development Cooperation Agency/Department for Research Cooperation (SIDA/SAREC) grant number SWE-2005-111 and the Danish International Development Cooperation (DANIDA).

Competing interests None.

Provenance and peer review Not commissioned; externally peer reviewed.

\section{REFERENCES}

1. World Health Organization. Guidance for National Tuberculosis Programmes on the Management of Tuberculosis in Children, WHO/HTM/TB/2006.371. Geneva: WHO, 2006.

2. Raviglione MC, Snider DE Jr, Kochi A. Global epidemiology of tuberculosis. Morbidity and mortality of a worldwide epidemic. JAMA 1995;273:220-6.

3. Hesseling AC, Schaaf HS, Gie RP, et al. A critical review of diagnostic approaches used in the diagnosis of childhood tuberculosis. Int J Tuberc Lung Dis 2002;6:1038-45.

4. Donald PR. Childhood tuberculosis: the hidden epidemic. Int J Tuberc Lung Dis 2004:8:627-9

5. Lienhardt C, Sillah J, Fielding $\mathrm{K}$, et al. Risk factors for tuberculosis infection in children in contact with infectious tuberculosis cases in the Gambia, West Africa. Pediatrics 2003;111:e608-14.

6. Gustafson $\mathbf{P}$, Lisse I, Gomes V, et al. Risk factors for positive tuberculin skin test in Guinea-Bissau. Epidemiology 2007;18:340-7.

7. Chapman JS, Dyerly MD. Social and other factors in intra-familial transmission of tuberculosis. Am Rev Respir Dis 1964;90:48-60.

8. Lockman S, Tappero JW, Kenyon TA, et al. Tuberculin reactivity in a pediatric population with high BCG vaccination coverage. Int J Tuberc Lung Dis 1999:3:23-30.

9. Young TK, Mirdad S. Determinants of tuberculin sensitivity in a child population covered by mass BCG vaccination. Tuberc Lung Dis 1992;73:94-100.

10. Davies PD. The natural history of tuberculosis in children. A study of child contacts in the Brompton Hospital Child Contact Clinic from 1930 to 1952. Tubercle 1961;42 (Suppl): $1-40$

11. Zeidberg L, Gass R, Dillon A, et al. The Williamson County Tuberculosis Study. A twenty-four-year epidemiologic study. Am Rev Respir Dis 1963;87:1-88.
12. Lewinsohn DA, Gennaro ML, Scholvinck L, et al. Tuberculosis immunology in children: diagnostic and therapeutic challenges and opportunities. Int J Tuberc Lung Dis 2004:8:658-74.

13. Gedde-Dahl T. Tuberculous infection in the light of tuberculin matriculation. $A m \mathrm{~J}$ Hyg 1952;56:139-214.

14. Chintu C, Mudenda V, Lucas S, et al. Lung diseases at necropsy in African children dying from respiratory illnesses: a descriptive necropsy study. Lancet 2002;360:985-90.

15. Walls T, Shingadia D. Global epidemiology of paediatric tuberculosis. J Infect 2004;48:13-22.

16. Starke JR. Pediatric tuberculosis: time for a new approach. Tuberculosis (Edinb) 2003:83:208-12

17. Gustafson P, Gomes VF, Vieira CS, et al. Tuberculosis in Bissau: incidence and risk factors in an urban community in sub-Saharan Africa. Int $\mathrm{J}$ Epidemiol 2004:33:163-72

18. Gustafson $\mathbf{P}$, Gomes VF, Vieira CS, et al. Tuberculosis mortality during a civil war in Guinea-Bissau. JAMA 2001;286:599-603.

19. Seng $\mathbf{R}$, Gustafson P, Gomes VF, et al. Community study of the relative impact of HIV1 and HIV2 on intrathoracic tuberculosis. AIDS 2002;16:1059-66.

20. Gustafson P, Gomes VF, Vieira CS, et al. Clinical predictors for death in HIV-positive and HIV-negative tuberculosis patients in Guinea-Bissau. Infection 2007;35:69-80.

21. Enarson DA, Rieder HL, Amadottir T, et al. Management of Tuberculosis. A Guide for Low Income Countries. 5th edn. Paris: International Union Against Tuberculosis and Lung Disease, 2000

22. Garly ML, Bale C, Martins CL, et al. BCG vaccination among West African infants is associated with less anergy to tuberculin and diphtheria-tetanus antigens. Vaccine 2001;20:468-74

23. Sodemann M, Rodrigues A, Nielsen J, et al. Maternal Vulnerability and Socio-economic Inequalities in Child Mortality. In: Indepth Network: Measuring Health Equity in Small Areas. London: Ashgate, 2005:87-108.

24. Sodemann M, Biai S, Jakobsen MS, et al. Knowing a medical doctor is associated with reduced mortality among sick children consulting a paediatric ward in Guinea-Bissau, West Africa. Trop Med Int Health 2006;11:1868-77.

25. Hershfield E. Tuberculosis in children. Guidelines for diagnosis, prevention and management (a statement of the scientific committees of the IUATLD). Bull Int Union Tuberc Lung Dis 1991;66:61-7.

26. Brailey ME. Prognosis in white and colored tuberculous children according to initia chest X-ray findings. Am J Public Health Nations Health 1943;33:343-52.

27. Pope AS, Sartwell PE, Zacks D. Development of tuberculosis in infected children. Am J Public Health Nations Health 1939;29:1318-25.

28. Grzybowski S, Barnett GD, Styblo K. Contacts of cases of active pulmonary tuberculosis. Bull Int Union Tuberc 1975:50:90-106.

29. Marais BJ, Gie RP, Schaaf HS, et al. The clinical epidemiology of childhood pulmonary tuberculosis: a critical review of literature from the pre-chemotherapy era. Int J Tuberc Lung Dis 2004:8:278-85.

30. Behr MA, Warren SA, Salamon H, et al. Transmission of Mycobacterium tuberculosis from patients smear-negative for acid-fast bacilli. Lancet 1999;353:444-9. 